\title{
Spray-dried liposomes: A study of the effect of carbohydrate carrier and concentrations on liposome size and drug entrapment
}

\begin{abstract}
Background and objective: Dry powder inhalation provide many promising features for drug delivery to the pulmonary system, such as localization of drug within the lungs, drug retention in the respiratory tract, and reduced systemic toxicities. This study aimed to prepare dry powders of liposomes containing salbutamol sulfate using spray drying and study the influence of carrier type and concentration on liposome size and drug entrapment following rehydration of liposomal powder.
\end{abstract}

Methods: Ethanol based proliposomes technique was used to generate liposomes containing salbutamol sulfate. Carbohydrate carriers: lactose, trehalose, sucrose, and mannitol in 1:6 or $1: 10(\mathrm{w} / \mathrm{w})$ lipid to carrier ratio were separately incorporated into liposome formulations and dried by Büchi Mini-SprayDryerB-290. The protective effects of the sugars were investigated in terms of product yield (\%), volume median diameter and size distribution of liposomes and entrapment efficiency of the drug.

Results: The product yield (\%) of spray dried liposome formulations with carriers was in the following order; trehalose $(94.02 \pm 1.20 \%)>$ sucrose $(69.91 \pm 1.75 \%)>$ lactose $(68.30$ $\pm 1.14 \%)>$ mannitol $(50.16 \pm 0.80 \%)$ for the $1: 6$ formulations. Depending on the carrier type and lipidto carrier ratio, the vesicle size of liposomes with lactose and mannitol increased significantly $(P<0.0001$ and $P=0.0027$, respectively), while the vesicle size with trehalose and sucrose varied to a lesser extent $(P>0.05)$ compared to the control formulations $(3.6 \mu \mathrm{m} \pm 0.24)$. For $1: 10$ formulation, the vesicle size with mannitol $(P<0.001)$ and sucrose $(P<0.001)$ increased significantly. The drug entrapment efficiency using the $1: 6$ formulations was $20.74 \pm 1.78 \%$ for sucrose, $35.59 \pm 2.42 \%$ for trehalose, $67.21 \pm 1.89 \%$ for lactose, and $80.84 \pm 3.64 \%$ for mannitol.

Conclusion: Stable spray-dried liposomes were manufactured using sugars as carriers. The findings of this study have demonstrated the potential using of carbohydrate carriers to increase the physical stability of liposomes during the drying process, and trehalose can offer formulations with most desirable characteristics, while mannitol showed the least benefits.

Keywords: Carbohydrate; Proliposome; Salbutamol sulfate; Spray drying.

\section{Introduction}

Liposomal formulations in the liquid state have been suggested for delivery to the lung to treat chronic pulmonary diseases like asthma and chronic obstructive pulmonary disease (COPD) using nebulizers. However, the stability of liposome vesicles in liquid formulations and leakages of the entrapped drug are the major issues during nebulization. ${ }^{1}$
Additionally, liposomes in liquid dispersions are chemically and physically unstable due to phospholipid hydrolysis and oxidation. Eventually, these chemical instabilities in phospholipid result in liposome aggregation and fusion with concomitant leakage of the originally encapsulated drug molecules. ${ }^{2}$ Drying technologies such as freeze-drying, spray-drying, spray-freezedrying, and supercritical fluid technology

${ }^{1}$ Department of Pharmaceutics, College of Pharmacy, Hawler Medical University, Erbil, I raq.

* Correspondence: nozad.hussein@hmu.edu.krd 
have all been investigated for the preparation of liposomesin the form of dry powders; hence promoting long-term storage stability of liposomes. ${ }^{3,4}$ Chennakesavulu and co-workers investigated the effectiveness of pulmonary delivery of colchicine and budesonide for idiopathic pulmonary fibrosis through designing a liposomal dry powder formulation. ${ }^{5}$ Among all drying technologies, spray drying is most widely used for the preparation of dry liposomesbecause it is a rapid technology for generating powdered formulations, and is relatively economical and convenient to use. ${ }^{4}$ However, the drying process may cause structural damage to the phospholipid membrane resulting in loss of the originally entrapped drug and low powder yielddue to material adherence to the internal walls of the spray-drier. ${ }^{6}$ Carries, such as mannitol, lactose, trehalose, and sucrose, act as stabilizers when added to liposome formulations prior to performing drying. Sugar scan be incorporated into the liposomal formulation to protect the liposomes from damage, and concomitant loss of the originally entrapped material during the dehydration process. Carbohydrates can also increase the production yield of the spray dried powder ${ }^{7}$ and ensure healthy reformation of vesicles upon rehydration of the spray-dried product. ${ }^{8}$ Omer and co-workers developed and investigated the liposomal dry powder formulation containing salbutamol sulfate using spray drying for respiratory delivery. They evaluated the effects of carrier type (lactose monohydrate or mannitol) and lipid to carrier ratio on the properties of the generated liposome. They found that spray drying can produce inhalable dry liposomes particles. ${ }^{9}$ Chougule and co-workers used spray drying to generate dried nanoliposome particles loaded dapsone using four different sugars, aimed to site specific delivery of dried liposomal encapsulated dapsone to the lung for prolonging drug retention at the site of application. ${ }^{10}$ This study aimed to investigate the effect of the inclusion of carbohydrate carriers and their concentration on the stability of liposomes, using salbutamol sulfate as a model drug. Four types of stabilizing carrier adjuvants (mannitol, trehalose, lactose, or sucrose) of two different concentrations were incorporated into the liposome formulations prior to spray drying. Powder yield (\%) and following rehydration investigations such as size, size distribution, zeta potential of the liposomes, and entrapment efficiency of the drug were all carried out.

\section{Methods}

\section{Design, setting and time of the study}

This experimental research study was carried out in both the College of Pharmacy, Hawler Medical University, and University of Central Lancashire, the UK from February to May 2014.

\section{Materials}

Soya phosphatidylcholine (Lipoid S-100; SPC) was supplied by Lipoid, Switzerland. Cholesterol (>99\%), D-(+) trehalose dehydrate, sucrose (>99\%), D-Mannitol $(>98 \%)$, and D-lactose monohydrate $(>98 \%)$ were obtained from Sigma Aldrich Ltd, UK. All HPLC grade solvents such as Absolute ethanol and HPLC-grade water were purchased from Fisher Scientific Ltd, UK. Salbutamol sulfate (also known as albuterol sulfate) was bought from Alfa Aesar, UK.

\section{Methods}

Liposome composition was adapted from the work conducted by Elhissi and co-workers, ${ }^{11}$ by using spray drying instead of the traditional rotary evaporators.

\section{Preparation of liposomes from proliposomes}

Lipid phase $(400 \mathrm{mg})$ consisting of cholesterol and SPC (1:4 mole ratio) was dissolved within a glass vial using $560 \mu \mathrm{L}$ of absolute ethanol at $70^{\circ} \mathrm{Cby}$ gentle manual mixing for approximately 3 minutes or until a clear solution of ethanol was obtained. Salbutamol sulfate solution $(4 \mathrm{ml} ; 10 \mathrm{mg} / \mathrm{ml})$ was added to the ethanolic solution of phospholipid and vortex mixed 
for 2 minutes to produce concentrated liposome dispersion (the primary hydration step). The solution was made up to $40 \mathrm{ml}$ using HPLC-grade water and shaken vigorously for an additional 2 minutes using a Whirl Mixer TM (Fisher Scientific, UK) to produces diluted liposomes $(10 \mathrm{mg} / \mathrm{ml})$; this hydration step was referred to 'secondary hydration.' Liposomes were left for 1 hour at room temperature to anneal. The sugar (lactose, mannitol, sucrose, or trehalose) was weighed and added to liposomal dispersion at 1:6 or1:10(w/w) lipid to carrier ratio.

\section{Spry-drying}

The Büchi Mini-SprayDryer B-290 (Büchi, Switzerland) was used to dehydrate the liposomes. During the drying process, the inlet temperature was set to $120^{\circ} \mathrm{C}$, aspirator rate to $100 \%$, spray gas flow to $\sim 8 \mathrm{~L} / \mathrm{min}$ and feed pump rate was set at $12 \mathrm{ml} / \mathrm{min}$, which provided an outlet temperature ranging from $65-85^{\circ} \mathrm{C}^{12}$

\section{Product yield}

The percentage of product yield of spray dried powder of various formulations was determined as the ratio of the amount of powder collected from the collecting vessel after every spray drying experiment to the total original weight of the carrier, drug, and lipids. The percentage of production yield was calculated according to the following equation:

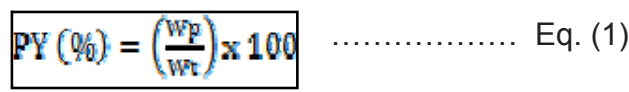

Where PY is the production yield; $W_{p}$ is the practical weight of spray-dried powder; and $W_{t}$ is the total theoretical weight of solid materials before spray-drying.

\section{Particle size analysis}

The size (volume median diameter, VMD) and SPAN of liposomes were measured using laser diffraction (Malvern Master sizer S; Malvern instruments Ltd, UK). Span $=(90 \%$ undersize $-10 \%$ undersize $) /$ VMD $(50 \%$ undersize $)$. To rehydrate

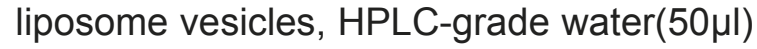

was added to spray dried powder $(10 \mathrm{mg})$ and vortex-mixed for 2 min to re-generate the liposomes in a liquid dispersion. The volume was made up to $1 \mathrm{ml}$ with HPLCgrade water. The dispersion was then left for 1 hour to anneal. Particle size analysis was then conducted. ${ }^{13}$

\section{Determination of drug entrapment efficiency}

To determine the entrapment efficiency (EE) of salbutamol sulfate, HPLC-grade water $(50 \mu \mathrm{l})$ was added to spray-dried powder $(10 \mathrm{mg})$ followed by vortex-mixing for 2 minutes to rehydrate the vesicles. The volume was made up to $1 \mathrm{ml}$ with HPLC-grade water, followed by annealing for 1 hour at room temperature. The dispersions were diluted to $8 \mathrm{~mL}$ with water, followed by centrifugation to separate the entrapped drug from the un-entrapped amount using a Beckman LM-80 ultracentrifuge (Beckman Coulter Instruments) at a speed of 55,000 rpm for $20 \mathrm{~min}$ at $6^{\circ} \mathrm{C}$. The supernatant was then collected and analyzed to determine the percentage proportion of unentrapped salbutamol sulfate using HPLC (Agilent 1200 with UV detector; Hewlett-Packard Co., USA). The entrapment efficiency (EE) was calculated using the formula:

$$
\mathrm{EE}(\%)=\left(\frac{\text { Total drug-Unentrapped drug }}{\text { Total Dray }}\right) \times 100 \quad \ldots \text { Eq. }
$$

\section{Statistical analysis}

All experiments were carried out in triplicate. The values were represented as means \pm standard deviation. Analysis of variance (ANOVA) and Student's t-test (paired t-test to compare similar groups before and after spray drying, whereas unpaired t-test between two independent groups) were used to determine the difference between samples. The variation was considered statistically significant when the $P$ value was less than 0.05 . The statistical analysis was performed using the statistical package for the social sciences software. 


\section{Results}

In this study, spray drying using different types of sugar was used to generate stable dry liposome powders. The results have shown that the inclusion of carbohydrates to liposome formulations prior to spray drying can promote the production yield, influence surface charge, improve entrapment efficiency, and reduce size and size distribution of the rehydrated liposomes.

Production yield of spray drying

Spray drying of liposomes in the absence of carbohydrates resulted in $0 \%$ yield due to adherence of the solid materials to the inner wall of the drying chamber. By contrast, the use of carbohydrates improved the yield. When 1:6 ratio $(\mathrm{w} / \mathrm{w})$ of lipid to carrier was used the percentage yield increased in the following order; Trehalose $(94.02 \pm 1.2)>$ sucrose $(69.91 \pm$ $1.75 \%)>$ lactose $(68.3 \pm 1.14 \%)>$ mannitol $(50.16 \pm 0.80 \%)$. However, for the formulation $1: 10$ ratio the percentage of yield was in the following order; Sucrose $(67.13 \pm 0.81)>$ lactose $(64.63 \pm 1.27)$ $>$ trehalose $(63.2 \pm 0.88)>$ Mannitol (34.63 $\pm 0.95)$ (Figure 1).

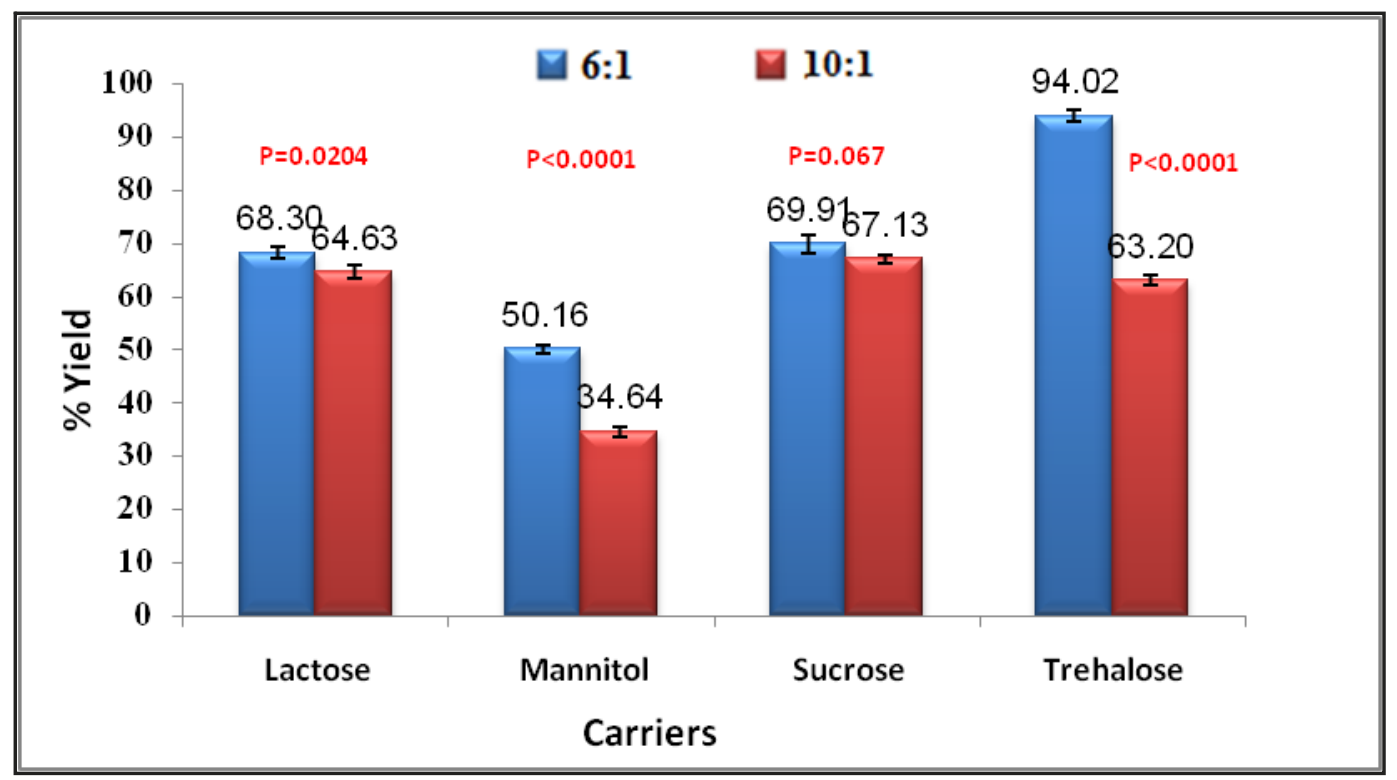

Figure 1: The effect of carrier type and concentration on spray drying yield. Spray drying conditions; temperature $120^{\circ} \mathrm{C}$, aspirator rate $100 \%$, gas flow $8 \mathrm{~L} / \mathrm{min}$, feed pump rate $12 \mathrm{ml} / \mathrm{min}$, and outlet temperature $65-85^{\circ} \mathrm{C}$. 


\section{Size and size distribution of the rehydrated vesicles}

The particle size of the liposome vesicles was measured before and after spray drying, and the effect of sugar type and concentration was investigated (Figure 2). The addition of carbohydrates had a significant effect on vesicle size. The size of liposome increased significantly with mannitol $(P<0.0001)$ and lactose $(P=0.0027)$ (1:6 mole formulations), while for formulation prepared with 1:10 lipid to carrier ratio, the vesicle size increased significantly with mannitol $(P=0.0004)$ and sucrose $(P=0.0031)$ compared to the size of freshly prepared liposomes and without inclusion of carbohydrates $(3.6 \mu \mathrm{m} \pm 0.04)$. The polydispersity of vesicles was measured before and after spray drying. The impact of carbohydrate carriers on the polydispersity of vesicles was not significant regardless of their size $(P<0.05)$, except for lactose $(P<0.01)$ compared to the polydispersity of fresh liposome formulation $(1.93 \pm 0.10)$ without carriers (Figure 3). Upon drying, the polydispersity was significantly changed for most of the carbohydrate carriers.

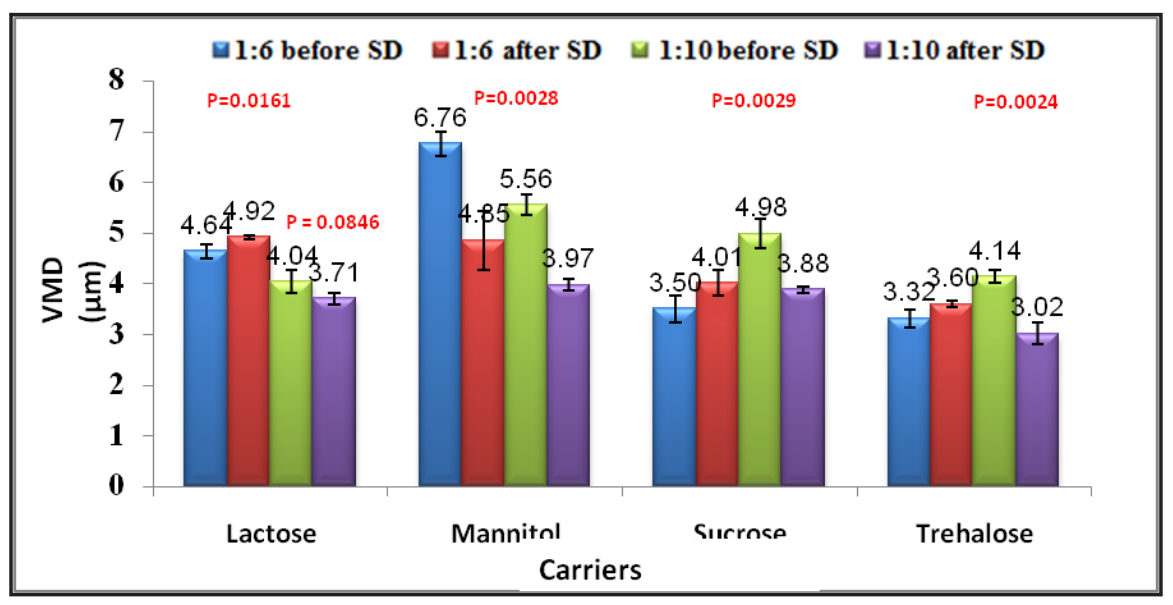

Figure 2: The effect of carrier type and concentration on liposomes size before and after spray drying (SD). Spray drying conditions; temperature $120^{\circ} \mathrm{C}$, aspirator rate $100 \%$, gas flow $8 \mathrm{~L} / \mathrm{min}$, feed pump rate $12 \mathrm{ml} / \mathrm{min}$, and outlet temperature $65-85^{\circ} \mathrm{C}$.

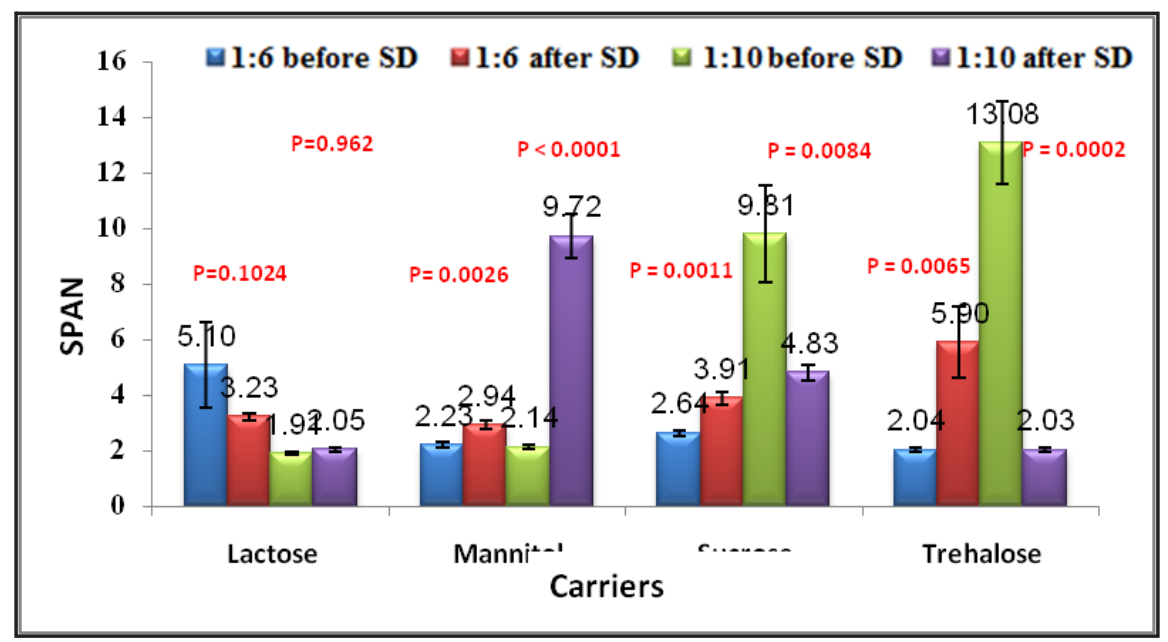

Figure 3: Polydispersity of liposomes produced before and after spray drying. Spray drying conditions; temperature $120^{\circ} \mathrm{C}$, aspirator rate $100 \%$, gas flow $8 \mathrm{~L} / \mathrm{min}$, feed pump rate $12 \mathrm{ml} / \mathrm{min}$, and outlet temperature $65-85^{\circ} \mathrm{C}$. 
The effects of carbohydrate carriers on entrapment efficiency

Following hydration of the spray-dried proliposome powders with HPLC water, the EE was measured, as shown in Figure 4. The EE were dependent on the type of carbohydrate carrier and lipid to carrier ratio used. The EE for the 1:6 formulations with sucrose, trehalose, lactose, or mannitol was $20.74 \%, 35.59 \%, 67.21 \%$, and $81.84 \%$, respectively. A decline in production yield was observed for both lactose and mannitol when the ratio of carrier to the lipid increased while increasing of trehalose ratio to the lipid concentration increased the entrapment efficiency of salbutamol sulfate significantly $(P<0.0001)$ compared to the lower ratio of carrier to lipid formulation.

\section{Discussion}

The effect of carbohydrate on powder yield

The drying process exerts a detrimental effect on liposome integrity and reduces production yield. Previous publications have highlighted a solution to this obstacle by using carbohydrates as a protective agent. $^{8,11}$ Since all the formulations were spray-dried using the same conditions, the difference in production yield is expected to be due to the lipid to sugar ratio and the type of sugar used. A previous study has reported a link between glass transition temperature $\left(T_{g}\right)$ and the type or concentration of carriers used. The material can become sticky if the carriers are dried above their $T_{g}$, resulting in adherence of most materials to the inner wall of the drying chamber. ${ }^{14,15}$ Therefore, there is no yield production of the powder formulations without carriers (i.e., yield $=$ $0 \%$ ). The production yield was increased when carbohydrate carriers added to the liposome formulation. The results are in accordance with those obtained by Omer, who studied the influence of carbohydrate carriers to the lipid ratio on the property of proliposomes generated from spray drier. ${ }^{16}$ Depending on the present findings, carriers with higher $\mathrm{T}_{\mathrm{g}}$ are expected to make the liposome preparations less sticky and more able during drying. Thus, the difference in the melting point of the carriers may lead to different production yields. The melting point of the carbohydrate adjuvants used was in the following order: trehalose (203 $\left.{ }^{\circ} \mathrm{C}\right)>$ lactose $\left(202.8^{\circ} \mathrm{C}\right)>$ sucrose $\left(186^{\circ} \mathrm{C}\right)$

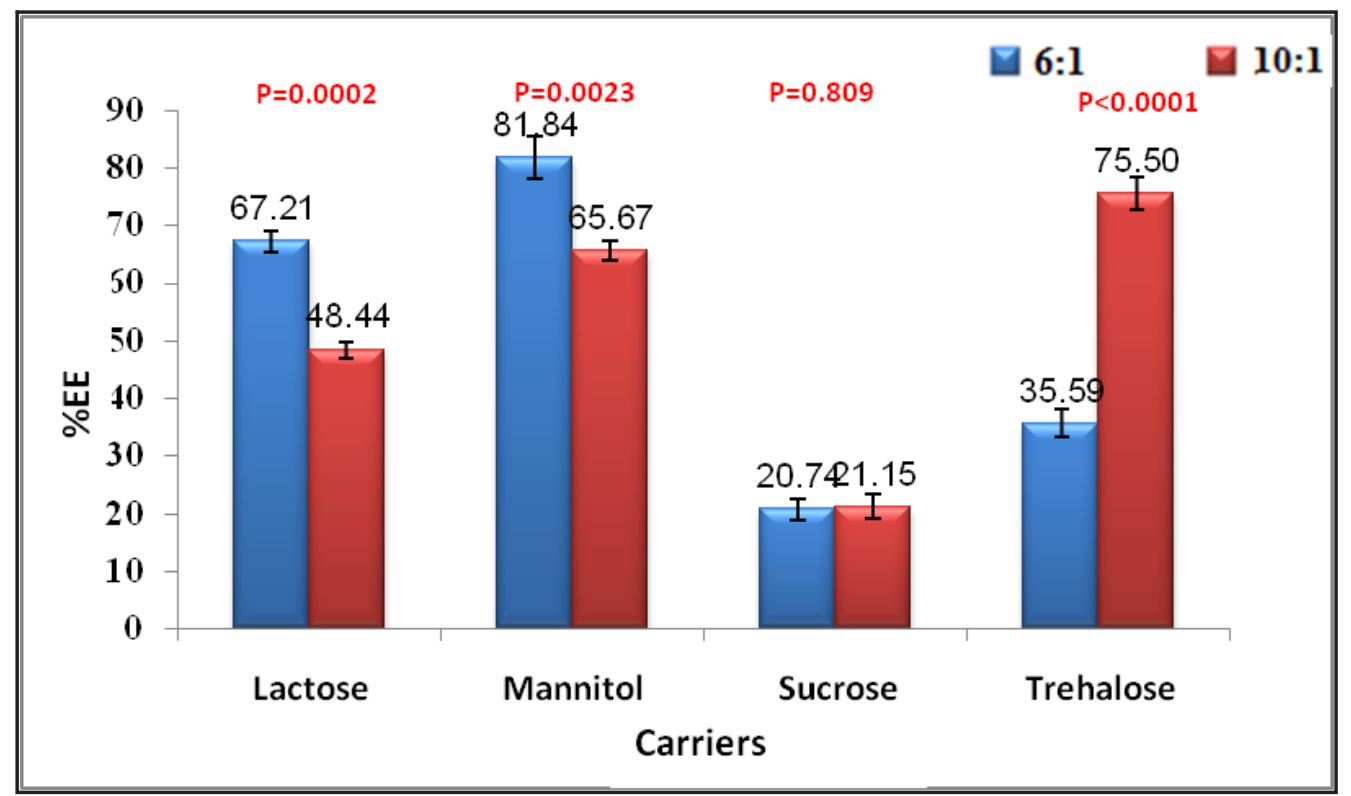

Figure 4: The effect of carrier type and concentration on drug entrapment efficiency following hydration of the spray dried liposome powders. 
>mannitol $\left(165-169{ }^{\circ} \mathrm{C}\right)$. This study demonstrated trehalose has the highest production yield $(94.02 \%)$, whereas the mannitol has the lowest yield $(50.16 \%)$. This result is in accordance with those obtained by Chen and co-workers, they investigated the effects of carbohydrate carriers on the spray dried liposome powder characteristics. ${ }^{8}$ The lower production yield of the mannitol-based powder is attributed to the lowest melting point of this sugar $\left(165-169^{\circ} \mathrm{C}\right)$, resulting in having highly sticky droplet components, which upon dehydration tended to melt and adhere to the internal walls of the drying chamber and subsequently low production yield was obtained. Regardless of carrier type, the production yield was decreased with increasing sugar concentration (Figure 1). Trehalose showed the greatest decrease $(P<0.0001)$, whereas sucrose showed less reduction in the yield. The decrease in production yield might be attributed to the susceptibility of the carriers to mechanical damage due to the transformation of the sugar into crystalline state and increasing adherence of sprayed droplets to the walls of the drying chamber. Additionally, the decline in the percentage yield production may also be related to thedesign of the spray dryer. Hence, dried parties were poorly collected by the cyclone. $^{14}$

\section{The effect of carriers on liposome size}

In pulmonary drug delivery applications, particle size is an important factor that determines the fate of inhaled material. For particles to possibly deposit into the deep lung (i.e., respiratory bronchioles and alveolar region), particles size needs to be less than $5 \mu \mathrm{m} .{ }^{17,18}$ The incorporation of carrier $(1: 6 \mathrm{w} / \mathrm{w})$ was significantly increased the VMD for lactose-based powder $(P=0.0027)$ and mannitol-based formulation $(P<0.0001)$, however no significant change in vesicle size was found for sucrose-based formulation $(P=0.6566)$ and trehalose-based powder $(P=0.1745)$, when compared to the control formulation (i.e., freshly prepared liposomes) $(3.6 \mu \mathrm{m}$ $\pm 0.24)$. Increasing carrier to lipid ratio from $1: 6$ to $1: 10$ weight ratio caused significant changes in liposome size for all formulations (Figure 2). The decrease in the size of mannitol and lactose based liposomes might be attributed to the internal alteration of the osmotic gradient. Thus, water has possibly migrated from the interior aqueous spaces of liposomes towards the continuous phase, causing shrinkage of the liposome vesicles. ${ }^{19}$ The increased liposome size for sucrose and trehalose might be attributed to the presence of the undissolved carrier. Thus, free sugar will be entrapped inside the matrix of the liposomes. Addition of water can cause rapid water movement from outside to inside the vesicles, ultimately increasing theliposome size, which also increased the entrapment efficiency of salbutamol sulfate. ${ }^{12}$

\section{Effects of spray drying conditions on liposomes}

For 1:6 lipid to carrier ratio, spray drying conditions did not change liposomal size for all sugars except for mannitol $(P=0.0062)$ the vesicle size was significantly decreased after spray drying (Figure 2). However, in formulations with higher sugar concentrations (i.e. 1:10 $(w / w)$ ), a significant effect on liposome size $(P<0.05)$ was observed for all sugars except lactose $(P=0.0846)$. The decrease in median liposome size was noted upon spray drying, for liposomes prepared with a 1:10 ratio; these findings coincide with the findings of Wessman et al. who studied the morphological changes of liposomes using spray drying and freeze drying. ${ }^{12}$ Generally, for formulation 1:6 weight ratio inclusion of sugars into the liposome formulations had a non-significant effect on the polydispersity of liposomes regardless of their size $(P>0.05)$, except for lactose $(P<0.01)$ compared to the polydispersity of fresh liposome formulation (1.93 \pm 0.10$)$ without carriers (Figure 3). Upon drying, an increase in polydispersity was noted for mannitol $(P=0.0026)$, sucrose $(P=$ $0.0011)$, and trehalose sugar $(P=0.0065)$, 
the greatest of which was seen for trehalose (3 fold increase). Furthermore, a significant change in the polydispersity of sucrose $(P=0.0021)$ and trehalose $(P=0.0002)$ was reported before spray drying when carrier concentration was increased to $1: 10$ compare with 1:6 lipid to sugar ratio. These findings indicate possible liposome aggregation. The drying process significantly decreased the polydispersity of both sucrose $(P=0.0084)$ and trehalose $(P=0.0002)$ based formulation, which may indicate the deaggregation of liposomes. In contrast, the polydispersity of the mannitol base formulation increased significantly $(P<0.0001)$ after spray drying (Figure 3$)$. The finding of this study is in agreement with those found by Chen et al. ${ }^{8}$ They explored both size and polydispersity index significantly increased for both sucrose and trehalose when they used in above optimum concentration.

\section{The effects of sugars on drug entrapment efficiency}

Figure 4 shows the entrapment efficiency (EE) of salbutamol sulfate in liposomes generated from mannitol, sucrose, lactose, or trehalose dried powders. The entrapment efficiency for the 1:6 formulations using sucrose, trehalose, lactose, and mannitol was $20.74 \%, 35.59 \%, 67.21 \%$, and $81.84 \%$, respectively. The variation in the entrapment efficiency of the drug in liposomes may be attributed to a number of factors, including liposome size, liposome bilayers and the influence of different types of sugars on the packing pattern of the liposome bilayers. Generally, increasing carrier concentration has a direct relationship with drug leakage from the vesicles. However, not all sugar carriers followed this trend. Liposomes with trehalose demonstrated the highest entrapment efficiency $(75.5 \%)$ at a higher ratio (1:10 formulation). A study conducted by Omer is in agreement with the finding of this experiment, whereby Omer found that an increase in carrier concentration can decrease in drug EE. ${ }^{16}$ Figures 2 and 4 show a link between size of the liposome and drug entrapment, as the carrier concentration increases a decrease in the VMD was noted for both lactose and mannitol sugar. The space within the liposome would decrease as VMD decreases, consequently reducing the space available for drug entrapment, which could explain the reduced EE showed. This may also be true for mannitol as it had the greatest size; therefore, it showed the greatest EE. Further studies using transmission electron microscopy can be carried out in the future to validate this hypothesis.

\section{Conclusion}

Spray drying of liposomes presented a viable way of producing high entrapment efficiency of stable liposome formulations using carbohydrates as a protectant. The addition of carbohydrate carriers had a significant effect on the characteristics of the produced liposomes. Spray drying reduced liposome aggregation, consequently reduced the measured size of liposomes for the formulation 1:10 lipid to carrier weight ratio. The results explored that the production yield (\%) of dried liposomes containing salbutamol sulfate with trehalose is the highest and with the smallest vesicle size. Spray drying has been demonstrated to be a successful process in the development of the liposomal powder and to preserve salbutamol sulfate in the formulation of SPC liposomes with trehalose.

\section{Competing interests}

The author declares no competing interests.

\section{References}

1. Rudokas M, Najlah M, Alhnan MA, Elhissi A. Liposome delivery systems for inhalation: A critical review highlighting formulation issues and anticancer applications. Med Princ Pract 2016; 25(Suppl 2):60-72.

2. Yadav AV, Murthy MS, Shete AS, Sfurti S. Stability Aspects of liposomes. Indian J Pharm Res Educ 2011; 45(4):402-13. 
3. Patel G, Chougule M, Singh M, Misra A. Nanoliposomal dry powder formulations. Method Enzymol 2009; 464:167-91.

4. Ingvarsson PT, Yang M, Nielsen HM, Rantanen J, Foged C. Stabilization of liposomes during drying. Expert Opin Drug Deliv 2011; 8(3):37588.

5. Chennakesavulu S, Mishra A, Sudheer A, Sowmya C, Suryaprakash Reddy C, Bhargav E. Pulmonary delivery of liposomal dry powder inhaler formulation for effective treatment of idiopathic pulmonary fibrosis. Asian J Pharm Sci 2018; 13(1):91-100.

6. Alexopoulou E, Georgopoulos A, Kagkadis KA, Demetzos C. Preparation and characterization of lyophilized liposomes with incorporated quercetin. J Liposome Res 2006; 16(1):17-25.

7. Samborska K, Gajek P, Kamińska-Dwórznicka A. Spray Drying of Honey: The Effect of drying agents on powder properties. Pol J Food Nutr Sci 2015; 65(2):109-18.

8. Chen K-H, Mueannoom W, Gaisford S, Kett VL. Investigation into the effect of varying I-leucine concentration on the product characteristics of spray-dried liposome powders. J Pharm Pharmacol 2012; 64(10):1412-24.

9. Omer HK, Hussein NR, Ferraz A, Najlah M, Ahmed W, Taylor KMG, et al. Spray-dried proliposome microparticles for high-performance aerosol delivery using a monodose powder inhaler. AAPS Pharm Sci Tech 2018; 19(5):2434 $-48$.

10. Chougule M, Padhi B, Misra A. Development of spray dried liposomal dry powder inhaler of dapsone. AAPS Pharm Sci Tech 2008; 9(1):4753.

11. Elhissi AMA, Karnam KK, Danesh-Azari M-R, Gill $\mathrm{HS}$, Taylor KMG. Formulations generated from ethanol-based proliposomes for delivery via medical nebulizers. J Pharm Pharmacol 2006; 58(7):887-94.

12. Wessman P, Edwards K, Mahlin D. Structural effects caused by spray- and freeze-drying of liposomes and bilayer disks. J Pharm Sci 2010; 99(4):2032-48.

13. Elhissi AMA, Islam MA, Arafat B, Taylor M, Ahmed W. Development and characterisation of freeze-dried liposomes containing two anti-asthma drugs. IET Micro Nano Lett 2010; 5(3):184-8.

14. Maury M, Murphy K, Kumar S, Shi L, Lee G. Effect of process variables on the powder yield of spray dried trehalose on a laboratory spray-drier. Eur J Pharm Biopharm Off J Arbeitsgemeinschaft Für Pharm Verfahrenstechnik EV 2005; 59:56573.

15. Muzaffar K, Nayik GA, Kumar P. Stickiness problem associated with spray drying of sugar and acid rich foods: A Mini Review. J Nutr Food Sci 2015; S12:S12003.
16. Omer H. Spray-dried bioadhesive formulations for pulmonary delivery [PhD Pharmaceutics]. [UK]: University of Central Lancashire; 2014.

17. Hupfeld S, Holsaeter AM, Skar M, Frantzen CB, Brandl M. Liposome size analysis by dynamic/ static light scattering upon size exclusion-/field flow-fractionation. J Nanosci Nanotechnol 2006; 6(9-10):3025-31.

18. Larhrib H, Martin GP, Marriott C, Prime D. The influence of carrier and drug morphology on drug delivery from dry powder formulations. Int $\mathrm{J}$ Pharm 2003; 257(1-2):283-96.

19. Alves GP, Santana MHA. Phospholipid dry powders produced by spray drying processing: structural, thermodynamic and physical properties. Powder Technol 2004; 145(2):13948. 